\title{
Simulation Research of Magnetically-coupled Resonant Wireless Power Transfer System with Single Intermediate Coil Resonator Based on S Parameters Using ANSYS
}

\author{
Liu Cheng, Peng Yan-qinga , Zhang Ji, Jiang Lian-ming, Tang Jia-cheng \\ School of Electrical Engineering and Automation, Xiamen University of Technology, Xiamen 361024, China
}

\begin{abstract}
ANSYS can be a powerful tool to simulate the process of energy exchange in magnetically-coupled resonant wireless power transfer system. In this work, the MCR-WPT system with single intermediate coil resonator is simulated and researched based on scattering parameters using ANSYS Electromagnetics. The change rule of power transfer efficiency is reflected intuitively through the scattering parameters. A new method of calculating the coupling coefficient is proposed. A cascaded 2-port network model using scattering parameters is adopted to research the efficiency of transmission. By changing the relative position and the number of turns of the intermediate coil, we find some factors affecting the efficiency of transmission. Methods and principles of designing the MCR-WPT system with single intermediate coil resonator are obtained. And these methods have practical value with design and optimization of system efficiency.
\end{abstract}

\section{Introduction}

Wireless power transfer (WPT) has become a research interest in recent years. It was reported by Tesla a century ago $^{[1]}$, and offers the promise of cutting the last wire. It has been or will be used in costly, inconvenient, dangerous and even some occasions that cannot use wired transmission, such as implantable ventricular assistant devices ${ }^{[2]}$ or for acquiring power from offshore wind driven generators and outer space solar power station ${ }^{[3]}$.

In 2007, the Massachusetts Institute of Technology (MIT) has proposed a new scheme based on strongly coupled magnetic resonances, thus presenting a key breakthrough for mid-range wireless energy transmission ${ }^{[4]}$. Although the coupled mode theory can explain the process of energy exchange well ${ }^{[5]}$, it can only apply to the conditions with small perturbations, and it is too hard for common electrical engineers to understand or use. So its application has been greatly restricted; on the other hand, measuring the parameters in the coupled mode theory is very difficult. In this work, we choose the ANSYS Electromagnetics, which is one of the most powerful tools to simulate the process of energy exchange in the MRCWPT system as well as to calculate the parameters in it, to study the WPT system with single intermediate coil resonator. From an electrical engineering perspective, we derive curves for coupling coefficient versus the gap length between the sending coil and receiving coil using ANSYS Hfss and the curve fitting tool of MATLAB, thus compare it with the result calculated by Neumann formula. Based on the above work, we research the influence of single intermediate coil with different number of turns on transmission efficiency. Paper ${ }^{[6]}$ has researched single intermediate coil resonator's impacts on transmission efficiency with different positions, but it ignores the geometrical factors of the intermediate coil. Paper ${ }^{[7]}$ has researched multiple intermediate resonant coils' influence on transmission efficiency, but it uses the coupled mode theory that many electrical engineers are not familiar with. By introducing the efficiency of transmission, a cascaded 2-port network model using $S$ parameters is adopted, which covers the principle of designing the single intermediate coil resonator and also avoids the blindness in designing the intermediate coil resonator.

\section{Modeling and Inductance Analysis}

\subsection{Simulation Model of Non-intermediate System}

As shown in Fig 1, the magnetic resonance coupling wireless power transfer system with non-intermediate resonant coil is composed of power supply, excitation coil, sending coil, receiving coil, load coil the load and for each coil there is one compensating capacitor installed in series. In this paper, only the efficiency of transferring energy from sending coil (labelled Coil 1) to receiving coil (labelled Coil 2) is considered, and for sake of simplicity, we suppose the load is a constant low resistive load which has no influence on the transmission efficiency analysis because each variable capacitor can tune the system to $2.8 \mathrm{MHz}$ that ensures all the coils are in resonance. The coil type in this analysis is planar spiral coil, and the 
number of turns of both sending coil and receiving coil is 7. The material of the coil is set to copper. Then the 3D model of the two coils is built in ANSYS and Fig 2 shows the model. The skin effect and proximity effect can be ignored in calculating the inductance for the following three reasons: firstly, the frequency of the system is far lower than the very high frequency (VHF); secondly, the wire diameter is not very thick; thirdly, the wire section is a uniform circle ${ }^{[8]}$.

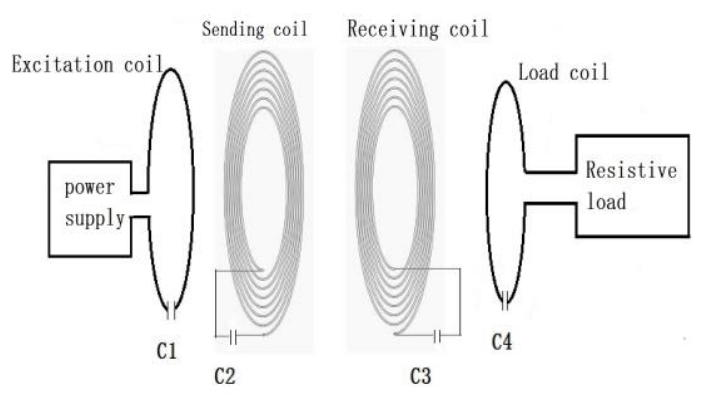

Fig 1. The MCR-WPT system with non-intermediate resonant coil.

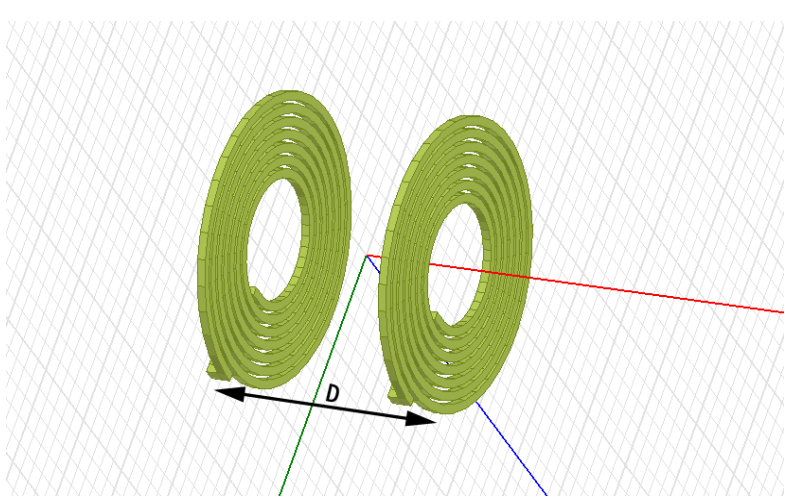

Fig 2. 3D model of sending coil and receiving coil.

\subsection{Calculating the Inductance and the Capacitor Value}

The inductance calculation of the coil is very important in the simulation, and the empirical formula for calculating the inductance of the planar spiral coil is given by equation (1) and the substitution is in equation (2), which indicates the relationship between the inductance value and its geometric size. The unit of the coil's size is inch and the unit of $\mathrm{L}$ (inductance) is $\mu H$. In order to make the meaning of the formulas more clear, all the coil sizes are labelled in Fig 3, which is the cutaway view of a common four-turn coil.

$$
\begin{gathered}
L=\frac{N^{2} \cdot A^{2}}{30 A-11 D_{I}} \\
A=\frac{D_{I}+N(W+S)}{2}
\end{gathered}
$$

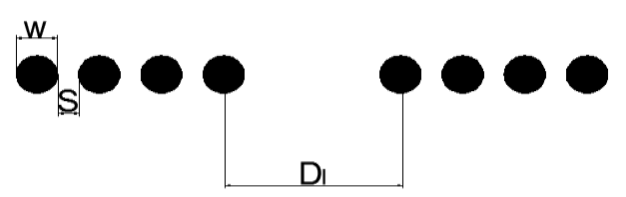

Fig 3. The cutaway view of a common four-turn coil.

In equation (1) and equation (2), $W, S, D_{I}$ and $N$ represent the wire diameter, the distance between two turns, inner diameter of the coil and the number of turns of the coil, respectively. In this paper, experimental parameters $W=11.8, N=7, W=0.6, S=0.31$, and the unit "inch" is for all the coil sizes. The theoretical result, as obtained from equation (1) and (2), and the simulation result, as obtained from ANSYS simulation, are shown and compared in Fig 4.

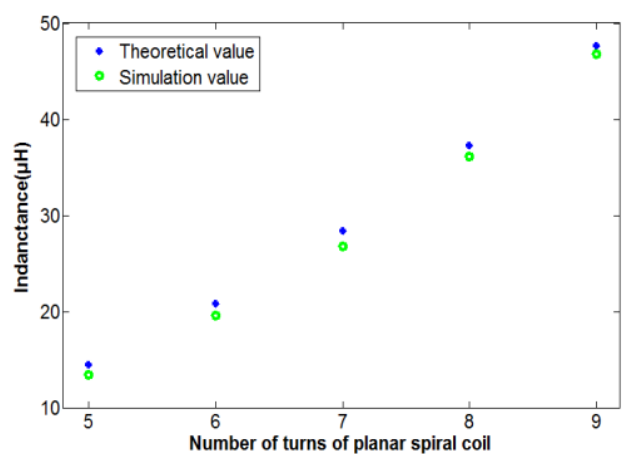

Fig 4. Results of the inductance calculated with two methods.

It can be seen from Fig. 4 that the two results have only minor differences in calculating the inductance. But the method of simulation will be better than that using theoretical formula when the shape of the coil is irregular. The simulation values of the Coil 1 and Coil 2 are $27.3 \mu \mathrm{H}$ and $27.1 \mu H$, respectively.

When the two coils are in resonance, the value of the compensating capacitor can be given by equation (3), where $f$ represents the resonant frequency.

$$
C=\frac{1}{4 \pi^{2} f^{2} L}
$$

The value of the capacitor for each coil $118.348 \mathrm{pF}$ and $119.22 \mathrm{pF}$. And the characteristic impedance of each coil is set to $50 \mathrm{ohm}$ for better impedance matching.

\subsection{Calculating the Coupling Coefficient between Resonators}

The scattering parameters are useful in analyzing the forward gain of the mid-range wireless systems.

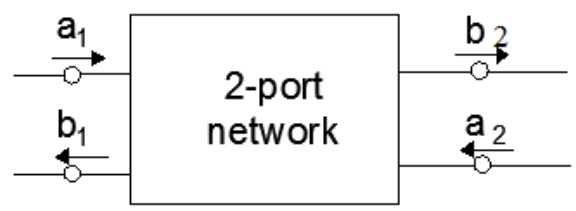

Fig 5. Sketch of a two-port network. 
As shown in Fig 5, the relationship of the incident and reflected waves can be defined by equation (4),

$$
\left[\begin{array}{l}
b_{1} \\
b_{2}
\end{array}\right]=\left[\begin{array}{ll}
S_{11} & S_{12} \\
S_{21} & S_{22}
\end{array}\right]\left[\begin{array}{l}
a_{1} \\
a_{2}
\end{array}\right]
$$

where $a_{1}$ and $a_{2}$ are the incident power waves on Port1 and Port-2, respectively; $b_{1}$ and $b_{2}$ are the reflected power waves on Port-1 and Port-2, respectively ${ }^{[9]}$. The forward transmission coefficient $S_{21}$ is defined as

$$
S_{21}=\frac{b_{2}}{b_{1}}=\frac{V_{2}^{-}}{V_{1}^{+}}
$$

It can be seen from equation (5) that the $S_{21}$ parameter can be an indicator for transmission performance of midrange wireless power systems. ANSYS Hfss(High frequency structure simulator) can calculate the $S$ parameters easily when the boundaries and excitations are arranged properly. Fig. 6 and Fig.7 show the amplitude and phase curves of $S_{21}$, respectively. And the solid lines with different markers stand for the $S_{21}$ parameters in different gap length between the two coils.

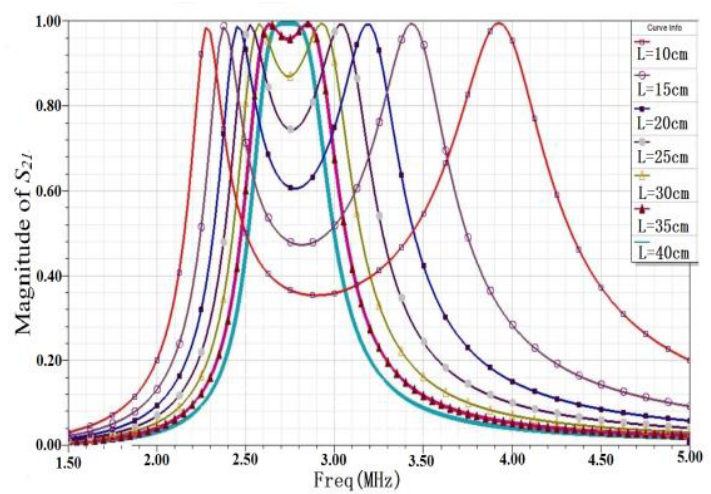

Fig 6. The amplitude curve of $S_{2 I}$.

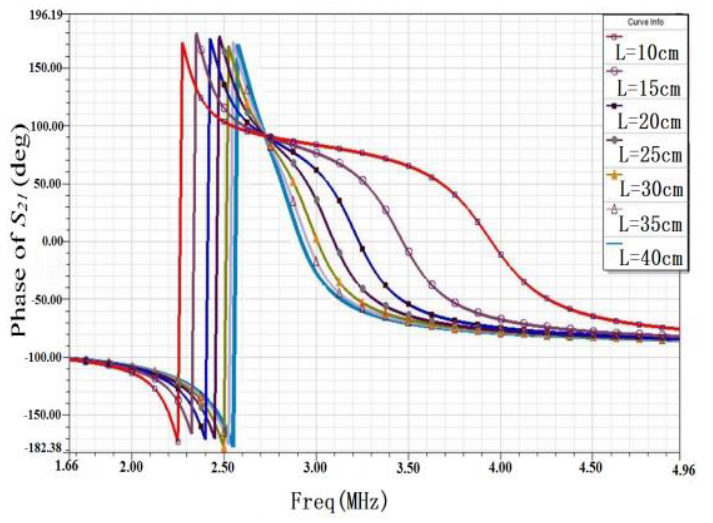

Fig 7.The phase curve of $S_{21}$.

It can be seen from Fig. 6 that there are two resonant peaks when the gap between the two coils varying from 10 centimeters to 35 centimeters with the step 5 centimeters. This phenomenon is so called "frequency splitting" what has been explained in great detail in paper $^{[10]}$, and two coils are said to be over-coupled. And when the gap is 40 centimeters, the two resonant peaks combine with each other and the system is said to be critical coupled.
Paper ${ }^{[11]}$ has proposed a formula to calculate the coupling coefficient between resonators based on $S$ parameters when the two resonators are over coupled, and it is given by equation (6).

$$
k=\sqrt{\frac{1}{2}\left[\left(\frac{f_{2}-f_{1}}{f_{0}}\right)^{2}+\left(\frac{f_{2}^{\prime}-f_{1}^{\prime}}{f_{0}^{\prime}}\right)^{2}\right]}
$$

where $f_{1}, f_{2}$ and $f_{0}$ represent the frequency of the left peak, the right peak and the minimum point in Fig.6, respectively and $f_{1}^{\prime}, f_{2}^{\prime}$, and $f^{\prime}{ }_{0}$ represent the frequency of the $\pm 180^{\circ}$ jump point, the $+90^{\circ}$ point and the zerocrossing point in Fig.7, respectively. But with increasing distance, the two resonators will be critical coupled and even under coupled, thus the equation (6) will fail to finish the calculation. In this condition, a fitting expression like equation (7) is proposed. And $a, b, c$ are deduced by the coupling coefficient $(D=0.35 \mathrm{~m}, 0.3 \mathrm{~m}, 0.25 \mathrm{~m}, 0.2 \mathrm{~m})$ obtained from equation (6) using the curve fitting tools of MATLAB. After the calculation, $a=0.037, b=-1.341$, and $c=-0.035$ are obtained.

$$
f(x)=a x^{b}+c
$$

Another method of calculating coupling coefficient of the two resonators is to use Neumann formula (8) and equation (9).

$$
\begin{gathered}
M=\frac{\mu_{0}}{4 \pi} \oint_{C_{1} C_{2}} \oint_{\frac{d l_{1}}{} \bullet d l_{2}} \\
k=\frac{M}{\sqrt{L_{1} L_{2}}}
\end{gathered}
$$

In equation (8), $\mathrm{M}$ and $\mathrm{D}$ represent the mutual inductance and the gap between the two coils, respectively. L1 and L2 represent the self-inductance of Coil 1 and Coil 2. Using equation (8) and equation (9) we can derive equation (10),

$k=\frac{\mu_{0} N_{1} N_{2}}{4 \pi \sqrt{L_{1} L_{2}}} \int_{0}^{2 \pi} \int_{0}^{2 \pi} \frac{r^{2} \cos \left(\theta_{1}-\theta_{2}\right)}{\sqrt{2 r^{2}-2 r^{2} \cos \left(\theta_{1}-\theta_{2}\right)+\mathrm{D}^{2}}} d \theta_{1} d \theta_{2}$

where $r$ is the average radius of the coils, and $r=0.242 \mathrm{~m}$ in this paper. Fig 8 shows and compares the results of coupling coefficient $k$ versus gap length between the two coils using different methods.

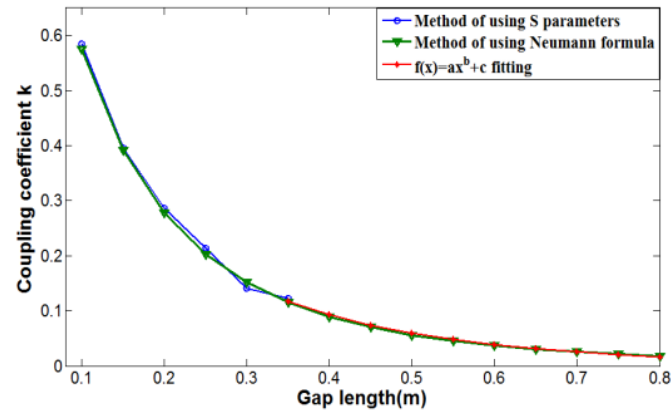

Fig 8. Coupling coefficient $k$ vs. gap length. 
The above simulation and calculation show that the results obtained from equation(6) is similar to that obtained from Neumann formula when the two coils are over coupled and the results obtained from the fitting expression(7) is similar to the one obtained from Neumann formula when the two coils are critical coupled or under coupled ( $D \in[0.35 m, 0.8 m]$ ). Paper ${ }^{[12]}$ uses the Method of Moments (MoM) to calculate coupling coefficient $k$ whose results are the same as the one obtained from Neumann formula. All the above methods are valid, and our method is maneuverable and suitable for engineering applications when the system is critical coupled or under coupled while using Neumann formula or MoM needs lots of mathematical techniques.

\section{Eficiency Analysis of WPT}

According to the microwave theory, the relationship between $S$ parameters and efficiency of transmission is given by equation (11).

$$
\eta_{21}=\left|S_{21}\right|^{2} \times 100[\%]
$$

It can be seen from Fig.6 and equation (11) that efficiency of transmission will decrease rapidly when the gap length between the two coils increases. In order to solve this problem, wireless power transfer system with single intermediate coil resonator was proposed in paper ${ }^{[13]}$.

When the gap length is 100 centimeters, the efficiency of transmission is lower than $8 \%$ and the coupling coefficient $k_{12}$ is so tiny that can be ignored. And in this condition, one intermediate coil resonator (labelled Coil 3) is placed in the middle of two coils.

Efficiency of transmission of the system with three coils can be deduced from a cascaded 2-port network model, what is showed in Fig 9.

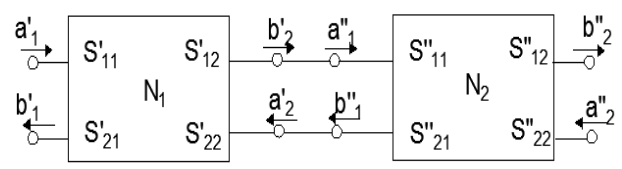

Fig 9. Sketch of a cascaded 2-port network.

$T$ parameters of the cascaded 2-port network model can be given by equation (12),

$$
T=T \cdot T^{\prime}
$$

where $T$ is the parameters of the first 2-port network $\mathrm{N}_{1}$ and $T^{\prime}$ is the parameters of the second one $\mathrm{N}_{2}$. And the relationship of $S$ parameters and $T$ parameters can be given by equation (13), and equation (14).

$$
\left[\begin{array}{ll}
S_{11} & S_{12} \\
S_{21} & S_{22}
\end{array}\right]=\frac{1}{T_{22}}\left[\begin{array}{cc}
T_{11} & T_{11} T_{22}-T_{12} T_{21} \\
1 & -T_{21}
\end{array}\right]
$$

$$
\left[\begin{array}{ll}
T_{11} & T_{12} \\
T_{21} & T_{22}
\end{array}\right]=\frac{1}{S_{21}}\left[\begin{array}{cc}
S_{12} S_{21}-S_{11} S_{22} & S_{11} \\
-S_{21} & 1
\end{array}\right]
$$

\subsection{Transverse Offset's Impact on Efficiency of Transmission}

The position of intermediate coil resonator's transverse offset is shown in Fig 10. And all the three coils have the same number of turns 7 .

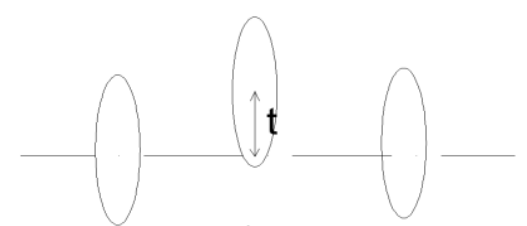

Fig 10. Diagrammatic sketch of intermediate coil's transverse offset

After the simulations and calculations, the relationship of maximum efficiency and intermediate coil resonator transverse offset can be seen in Fig 11.

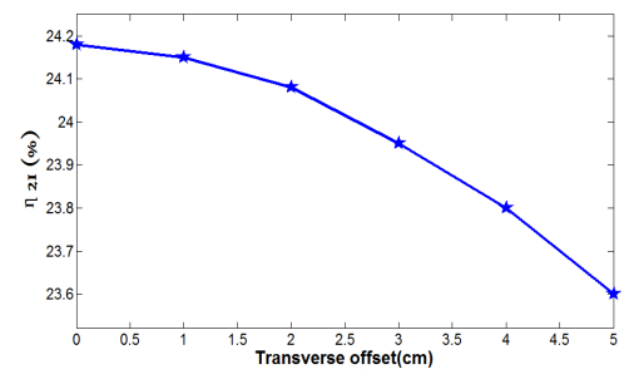

Fig 11. Maximum efficiency of transmission vs. transverse offset.

Fig 11 shows that small transverse offset of Coil 3 has minimal difference on the efficiency of transmission $\eta_{21}$. When designing the WPT system with single intermediate coil resonator, it's flexible for us to place the intermediate coil, instead of considering the small transverse offset of the intermediate coil.

\subsection{Axial Offset's Impact on Efficiency of Transmission}

The position of intermediate coil resonator's axial offset is shown in Fig 12. And all the three coils have the same number of turns 7 .

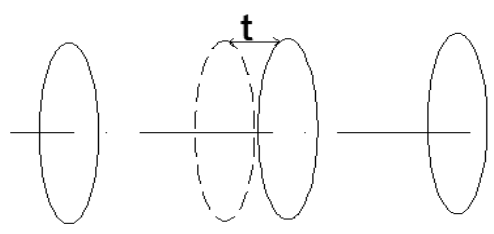

Fig 12. Diagrammatic sketch of intermediate coil's axial offset. 


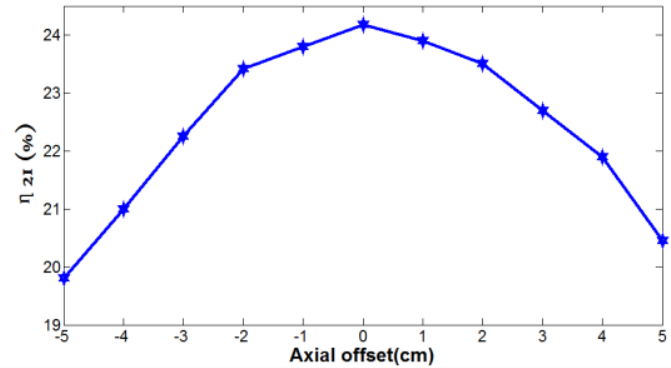

Fig 13. Maximum efficiency of transmission vs. axial offset.

Fig 13 shows the relationship of maximum efficiency and axial offset of Coil 3. Axial offset has significant effect on the efficiency of transmission. When designing the MRC-WPT system, we should place the intermediate coil resonator in the middle of the two coils to the greatest extent.

\subsection{Efficiency of Intermediate Coil in Different Turns}

In this simulation, the coil type of Coil 3 is the planar spiral coil and is also placed in the middle of Coil 1 and Coil 2. The number of turns varies from 5 to 9 whose inductance value has been calculated in section II .

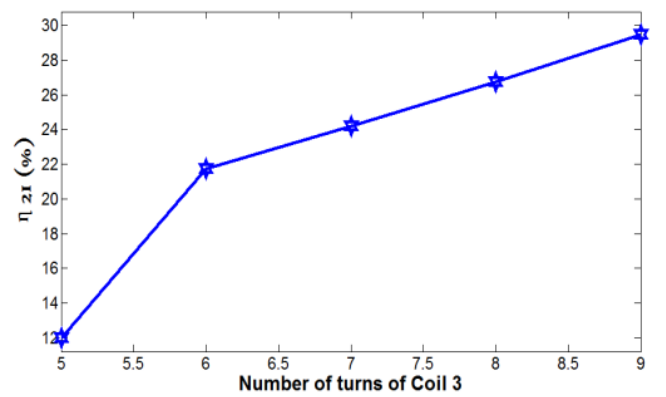

Fig 14. Maximum efficiency of transmission vs. number of turns of Coil 3.

With the increase in the number of turns, the inductance and radius of Coil 3 rise. As shown in Fig.14, higher efficiency of transmission can be obtained from increasing the number of turns of the intermediate coil. However, in the actual situation, radius of intermediate coil cannot be very large. And when the radius reach to some of extent, the resistance will be very large which can suppress the transmission efficiency ${ }^{[14]}$.

\section{CONCLUSION}

The self-inductance of coils can be calculated accurately in ANSYS Electromagnetics. And the method of calculating coupling coefficient using scattering parameters is developed, and thus a fitting expression like $f(x)=a x^{b}+c$ is proposed to calculate the coupling coefficient. When the two coils are critical coupled or under coupled, this method is easy and useful in engineering application.
Based on the above work, magnetically-coupled resonant wireless power transfer system with single intermediate coil resonator is simulated and researched using scattering parameters. A cascaded 2-port network model is used to explain the above system. Simulations and calculations show that transmission efficiency of the system is relative to intermediate coil's axial offset while intermediate coil's small transverse offset has minimal difference on the efficiency of transmission. When designing the system, we can call for the powerful tool-ANSYS Electromagnetics to determine the proper number of turns and radius of intermediate coil.

\section{ACKNOWLEDGMENT}

The authors would like to thank Prof. Chen Li-an for useful communications and discussions. And the authors gratefully acknowledge the help of School of Electrical Engineering and Automation, Xiamen University of Technology for powerful computers. This paper is supported by Project of Xiamen Municipal Bureau of Science (Grant No. 3502Z20153016), Graduates' Science and Technology Innovation Project of Xiamen University of Technology (Grant No. YKJCX2015001), the Highlevel Personal Project of Xiamen University of Technology (Grant No. KJ14005R).

\section{References}

1. J.P. Barrett, Electricity at the columbian exposition (Madison: R R Donnelley, 1894)

2. Y. Zhou, X.J. Yu, Trans. of CES, 30,103(2015)

3. N. Shinohara, S. Kawasaki, IEEE RWS, 13(2009)

4. A. Kurs, A. Karalis, R. Moffatt, J. D. Joannopoulos, P. Fisher, M. Soljačić, Sci., 317, 83 (2007)

5. A. Karalis, J.D. Joannopoulos, M Soljačić, Ann. of Phys., 323, 34 (2008)

6. B. LUO, M.T. SHENG, S.C. WU, M. ZHANG, Proc. CSEE, 33, 171(2013)

7. Z.Q. LI, S.D. HUANG, M.S. YANG, X.F. YUAN, Trans. CES, 28, 35(2013)

8. K. Tarloff, Caiyitelin. Inductance calculation manual (Beijing, China: China Machine Press, 1992)

9. S.Y.R. Hui, W. Zhong, C.K. Lee. IEEE Trans. on Power Electron., 29, 4500(2014)

10. W.Q. Niu, W. Gu, J. X. Chu, Electron. Lett. , 48, 723(2012)

11. S.E. ZHANG, C. MA, W.J. CUI. J. of NCEPU, 39, 77(2012)

12. T. Imura, Y. Hori. IEEE Trans. on Ind. Electron., 58, 4746(2011)

13. R.E. Hamam, A. Karalis, J.D. Joannopoulos. Ann. of Phys., 324, 1783(2009)

14. Y. Zhai, Y. Sun, X. Dai, Proc. CSEE, 32, 155(2012) 